\title{
La formación clínica como piedra angular de los estudios de medicina
}

\author{
José L. Álvarez-Sala Walther, M. Asunción Nieto Barbero, Gema Rodríguez Trigo
}

\section{¿Es la formación clínica la piedra angular de los estudios de medicina?}

Una primera pregunta que cabe plantear es si, verdaderamente, la formación clínica es, como indica el título de este artículo, o no la piedra angular de los estudios de medicina. Evidentemente, la respuesta a esta pregunta tiene que ser afirmativa y ello, al menos, por dos tipos de razones diferentes. La primera razón podría catalogarse como de índole legal o dependiente de normativas y disposiciones. En efecto, en la historia reciente siempre ha existido, en todos los planes de estudio que han estado vigentes hasta la fecha, en cualquier país de nuestro entorno, una referencia expresa a la necesidad de la formación clínica en los estudios de medicina. Que duda cabe que esta referencia es ahora aún mucho mayor al haberse puesto en marcha, en estos últimos años, el denominado Espacio Europeo de Educación Superior (EEES). La segunda razón podría considerarse como constitutiva o consustancial con la propia medicina, es decir, ligada a la esencia misma de la profesión y al hecho de ser médico y de ejercer como tal.

No obstante, la contestación a la pregunta que se ha formulado también debe matizarse o, quizás mejor, completarse con dos aseveraciones adicionales, asimismo muy importantes. Por un lado, no debe olvidarse que los conocimientos teóricos son del todo imprescindibles, incluso siendo la formación clínica la piedra angular de los estudios de medicina. Por otra parte, la investigación también es fundamental $y$, sin duda, inherente a la medicina. A continuación se desarrollan brevemente estos cuatro conceptos.
Fundamentos legales actuales que establecen la necesidad de la formación clínica en los estudios de medicina

Varios textos legales, normativas y disposiciones de diverso rango, vigentes en el momento actual, respaldan la absoluta necesidad de la formación clínica en los estudios de medicina, así como el carácter decisivo e irrenunciable de esta formación en todos los planes que tengan como objetivo la habilitación para el ejercicio de la profesión de médico.

Así, por ejemplo, en el preámbulo del real decreto 1393/2007, del Ministerio de Educación y Ciencia, en el que se establece la ordenación de las enseñanzas universitarias oficiales, se señala que la nueva organización de la enseñanza en la universidad responde no sólo a un cambio estructural, sino que además debe impulsar una modificación en los métodos docentes, que han de centrar su objetivo en el proceso de aprendizaje del estudiante, proceso que debe insertarse en un contexto que se extiende a lo largo de la vida [1]. Del mismo modo se indica que los planes de estudios han de tener, en el centro de sus objetivos, la adquisición de competencias, ampliando, sin excluirlo, el tradicional enfoque basado en contenidos y horas lectivas, por lo que debe hacerse énfasis en los sistemas de aprendizaje de dichas competencias, así como en los procedimientos para evaluar su adquisición. Por ello, se insiste más adelante, los planes de estudio tienen que garantizar que los títulos acreditan la posesión de las competencias y de los conocimientos que resultan adecuados para el ejercicio profesional. Finalmente, el preámbulo termina
Servicio de Neumología. Hospital Clínico San Carlos. Facultad de Medicina. Universidad Complutense. Madrid, España.

E-mail

jlasw@separ.es

Agradecimientos Al profesor Felipe Rodríguez de Castro por su ayuda en la ideación de este artículo y por facilitar el acceso a las figuras y a algunas de las citas bibliográficas que se incluyen en el trabajo. 
afirmando que la finalidad de las enseñanzas de grado se cifra en obtener una formación general válida para el desempeño de la profesión, para la transmisión de la cultura y para la iniciación en actividades de investigación, todo lo cual ha de facilitar la movilidad de los alumnos [2].

El real decreto 1393/2007 antes citado [1] establece, además, en su artículo 9, que las enseñanzas de grado tienen como objetivo la obtención, por parte del estudiante, de una formación general que ha de orientarse a la preparación para el ejercicio de actividades de carácter profesional. Es más, en su artículo 12, se señala que los planes de estudios deben diseñarse, en todo caso, de manera que permitan alcanzar las competencias necesarias para llevar a cabo esa profesión. A tales efectos, la universidad ha de justificar la adecuación de sus planes de estudio a dichas condiciones.

Muy importante al respecto es también la resolución del 17 de diciembre de 2007, de la Secretaría de Estado de Universidades e Investigación, por la que se fijan las condiciones que deben tener los planes de estudio conducentes a la obtención de títulos que habilitan para el ejercicio de la profesión de médico [3]. Esta resolución indica, en su apartado 3.5, que estos planes deben garantizar, en todo caso, la adquisición de las competencias necesarias para ejercer la medicina, de acuerdo con lo regulado en la normativa aplicable [4].

En el mismo sentido, la Agencia Nacional de Evaluación de la Calidad y Acreditación (ANECA) ha refrendado varias veces, por ejemplo con ocasión de la aprobación del plan de estudios del grado de medicina presentado por la Universidad Complutense de Madrid y por otras universidades españolas, que los objetivos generales de estos estudios deben dirigirse a adquirir los conocimientos, las habilidades y las actitudes que se requieren para realizar el diagnóstico, formular el pronóstico e indicar el tratamiento de las enfermedades físicas y psíquicas más prevalentes, así como para orientar el de las poco frecuentes. También para ser capaz de desarrollar actividades de prevención y promoción de la salud, para conseguir una buena capacidad de comunicación con los pacientes y sus familias, para conocer los fundamentos científicos de la medicina, iniciándose en la práctica de la investigación, y para lograr estos objetivos de modo tal que el estudiante aprenda a ejercer su futura profesión de médico [5].

\section{Razones constitutivas o consustanciales que avalan la necesidad de la formación clínica en los estudios de medicina}

La práctica clínica, esto es, el ejercicio real de la medicina, es la razón de ser de la profesión médica. Para adquirirla o, lo que es lo mismo, para poder ser un médico en el mejor sentido de la palabra, por tanto competente y con capacidad para progresar de modo autónomo, es obvio que hay que aprenderla bien desde el comienzo, para luego poder cultivarla durante toda la vida. De este hecho depende, en esencia, el futuro desarrollo profesional del estudiante que ahora está aprendiendo. De ahí que pueda establecerse que, sin duda alguna, la formación clínica es algo consustancial con los estudios de medicina y que para llegar a ser médico es imprescindible que esa formación, que inicialmente ha de adquirirse como estudiante, debe ser no sólo la más adecuada, sino también la mejor posible. Sólo así puede consolidarse luego, cuando se es médico.

Las razones y argumentos que avalan y respaldan esta idea son numerosas y de todo tipo. Baste con recordar al respecto, con una perspectiva histórica, un conocido pasaje de Maimónides (1135-1204): 'Concédaseme la oportunidad de mejorar y extender mi adiestramiento, pues no existe ningún límite al conocimiento. Ayúdeseme a corregir y complementar mis defectos a medida que se ensancha la amplitud de la ciencia y sus horizontes día a día. Otórgueseme el valor de darme cuenta de mis errores diarios para que mañana pueda ver y comprender bajo una luz mejor lo que no pude comprender bajo la escasa luz de ayer'. Otros muchos pasajes y textos, de similar contenido e importancia, escritos por reconocidos médicos clínicos, clásicos o recientes, podrían también citarse al respecto.

\section{La formación clínica es la piedra angular, pero los conocimientos teóricos resultan imprescindibles}

Esta afirmación posiblemente no necesita defensa alguna. En cualquier caso, toda formación clínica precisa de unos conocimientos teóricos previos, rigurosos y bien fundados, que sirven para dar a dicha formación todo su sentido, su aplicabilidad y su utilidad en la práctica. De ahí que los planes de estudio de medicina deban establecer, en un razonado equilibrio, la justa y co- 


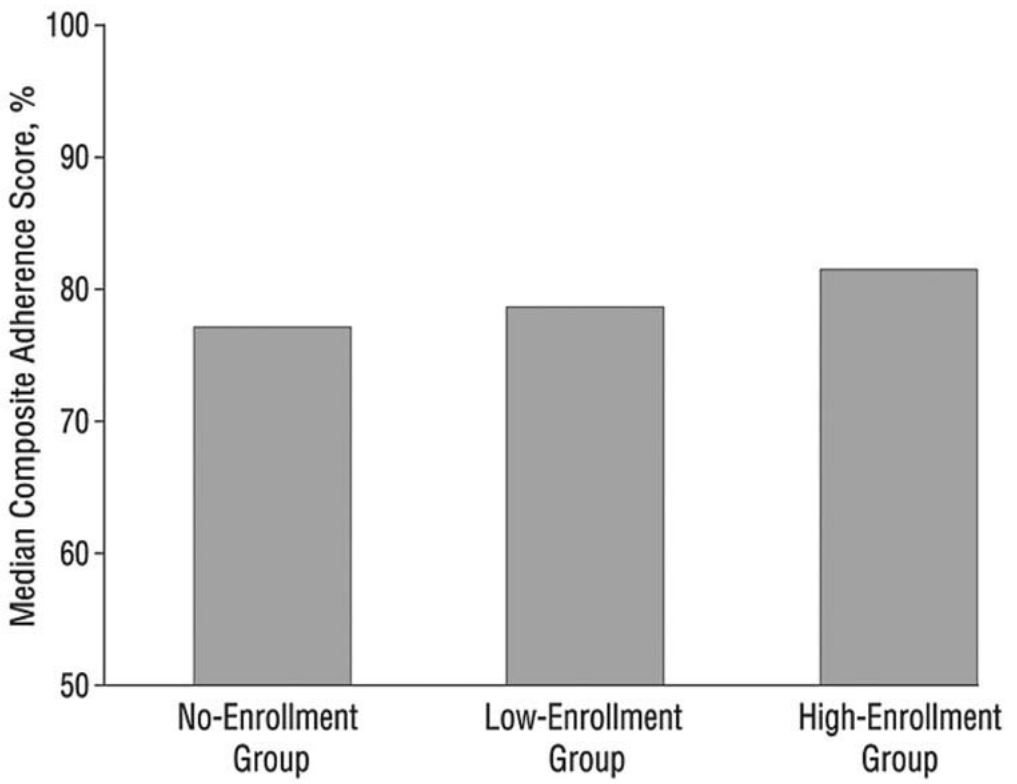

Figura 1. Cumplimiento de las recomendaciones diagnósticas y terapéuticas en 494 hospitales del estudio Crusade, estratificados en terciles según el grado de participación de esos hospitales en ensayos clínicos. Puede comprobarse que el cumplimiento de las recomendaciones es mayor en los hospitales en los que la participación en ensayos clínicos es superior. Tomado de [6].

rrecta proporción entre los contenidos teóricos y la formación clínica.

También en este caso cabe recordar conocidas frases de grandes clínicos que sirven para refrendar este aserto. Algunas pueden ser, por ejemplo, las dos siguientes: 'Sin la teoría, la práctica no es más que rutina provocada por la costumbre. Solamente la teoría puede producir y desarrollar el espíritu' (Louis Pasteur, 1822-1895) y 'Estudiar los fenómenos de la enfermedad sin libros es cruzar el mar sin cartas de navegación, pero estudiar libros sin ver enfermos no es ni tan siquiera embarcar' (William Osler, 1849-1919).

\section{La formación clínica es la piedra angular, pero la investigación es fundamental e inherente a la medicina}

Esta afirmación posiblemente tampoco necesita mucha argumentación. No hay duda de que la investigación mejora la actividad clínica y, por tanto, la práctica médica y la atención a los enfermos. En ello coinciden numerosos trabajos efectuados con distintos objetivos, diferentes variables y con diversas perspectivas. Recientemente, por ejemplo, Majumdar et al [6] han comprobado que el cumplimiento de las guías clínicas o de las recomendaciones diagnósticas y terapéuticas es más adecuado en los hospitales en los que la investigación clínica es mayor que en aquellos en los que apenas se realiza investigación alguna (Fig. 1). Y aún más importante, estos mismos autores [6] también han encontrado que la mortalidad a corto plazo, al llevar a cabo un determinado procedimiento terapéutico, es menor en los hospitales que están implicados en investigación clínica (Fig. 2).

Interesante al respecto es la encuesta de satisfacción sobre la calidad de la enseñanza recibida efectuada por Ríos-Zambudio et al [7] a 82 residentes de aparato digestivo que acababan de terminar su formación. Casi un $75 \%$ de los encuestados se mostraba altamente insatisfecho con la actividad investigadora que se había llevado a cabo, mientras que no llegaba a un $20 \%$ el porcentaje de residentes que refería estar conforme o muy conforme con lo que, en este sentido, se les había enseñado. Estos datos contrastaban con los hallados a propósito de la actividad asistencial desarrollada durante la residencia. Efectivamente, más de un $75 \%$ de los encuestados se mostraba satisfecho con la actividad y con las enseñanzas 


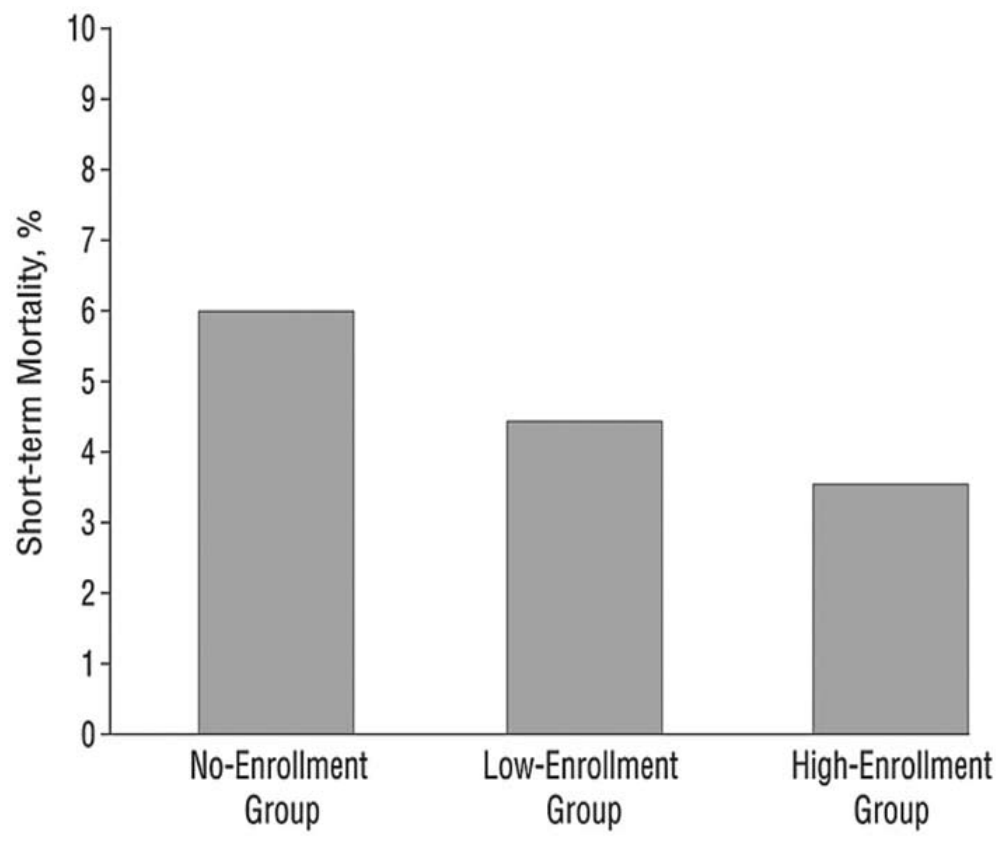

Figura 2. Mortalidad a corto plazo de 174.062 enfermos afectos de un síndrome coronario agudo tratados en 494 hospitales del estudio Crusade, estratificados en terciles según el grado de participación de esos hospitales en ensayos clínicos. Puede comprobarse que la mortalidad es menor en los hospitales en los que la participación en ensayos clínicos es mayor. Tomado de [6].

recibidas en este ámbito (Fig. 3). En ese mismo estudio pudo comprobarse que sólo dos de los 82 residentes había hecho la tesis doctoral durante su periodo de formación, mientras que $23 \mathrm{ni}$ siquiera se la habían planteado (Fig. 4) [7].

¿Pueden ponerse en relación los hechos mencionados, aunque incumban al posgrado y aunque sólo sea indiciariamente, con la escasa importancia que suele darse a la investigación en la carrera de medicina y con la poca expresión que suele concedérsele en los actuales planes de estudio? Quizás fuera conveniente meditar sobre este asunto y ello no sólo por su trascendencia intrínseca, es decir, por la importancia que la investigación tiene en la formación clínica de los estudiantes de medicina, sino también por la influencia que luego ejerce durante la residencia $\mathrm{y}$, en sentido más amplio, en el posgrado. Al respecto sería recomendable incluir en la enseñanza del grado (antes pregrado) el empleo, como herramienta en la clínica habitual, de la medicina basada en la evidencia. De este modo, los estudiantes podrían apreciar mucho mejor la importancia que tiene la investigación en la práctica asistencial.

\section{Requisitos que deben cumplirse para conseguir que la formación clínica sea adecuada}

La formación clínica de los estudiantes de medicina necesita, para que sea adecuada, unos requisitos previos que deben cumplirse rigurosamente. Al menos, los tres que se enumeran a continuación:

- Que el número de estudiantes sea el apropiado a las posibilidades docentes reales de cada facultad y que, por extensión, se ajuste a las verdaderas necesidades de médicos que tiene el país. En este sentido, el número de alumnos de nuevo ingreso en toda España ha pasado de 4.343 en el año 2005 a 5.821 en el año 2009. Puede afirmarse, por tanto, que las plazas que se han convocado para iniciar los estudios de medicina han crecido un $34 \%$ en los últimos tres años. A este hecho se suma la proliferación 'indiscriminada' de nuevas facultades, públicas y privadas, que se ha producido en este tiempo, lo que ha hecho que actualmente en España casi se haya alcanzado la cifra de 40 . Como 


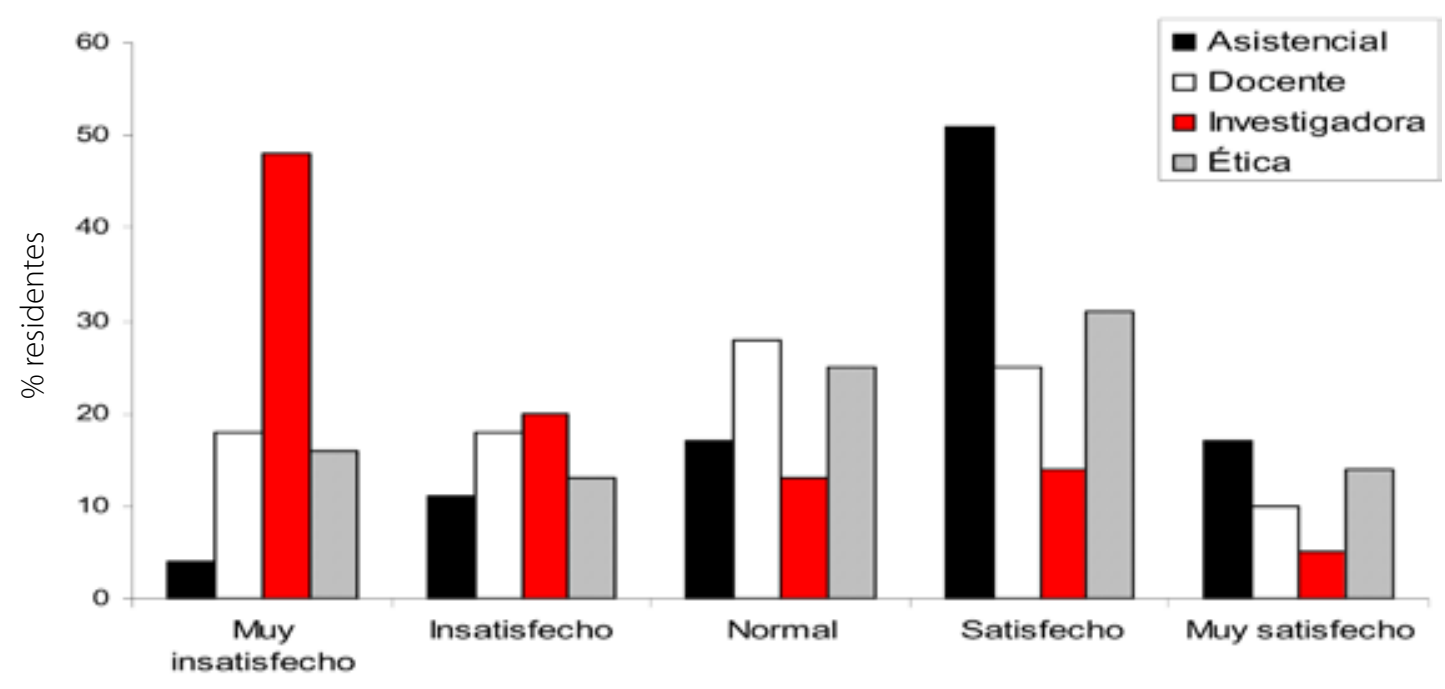

Figura 3. Encuesta de satisfacción, realizada a 82 residentes de aparato digestivo al terminar su periodo de formación, sobre la calidad de la enseñanza recibida durante la residencia. Puede observarse el alto grado de insatisfacción existente en cuanto a la actividad investigadora desarrollada, lo que contrasta con la alta satisfacción en lo referente a la actividad asistencial. Tomado de [7].

punto de referencia quizás merezca la pena recordar que el número de colegiados en nuestro país era de 165.358 en el año 1996 y fue de 219.031 en el año 2009 , lo que equivale a un aumento del $32 \%$ en 12 años. De esta forma, la tasa de médicos en España en el año 2009 alcanzó la cifra, según el Instituto Nacional de Estadística, de 480 médicos/100.000 habitantes (la segunda más alta de Europa), mientras que la media de la Unión Europea en el año 2005 era, según los datos de Eurostat, de 330 médicos/100.000 habitantes. Estos números hablan por sí solos y apoyan el punto de vista de la Conferencia Nacional de Decanos de Medicina, que es contraria a la idea, tan extendida en el momento actual en cierta administración sanitaria y entre algunos políticos del ramo, según la cual se defiende, más o menos y con evidente simpleza, que 'cuantos más medicos, mejor'.

- Que los estudiantes de medicina se integren verdaderamente en las actividades asistenciales de un hospital que pueda llamarse universitario con propiedad, en el que pueda llevarse a cabo una formación clínica apropiada y correcta. En este sentido cabe mencionar, por ejemplo, que en el plan de estudios del grado de medicina de la Universidad Complutense de Madrid, aprobado el 8 de mayo de 2009 por la ANECA, se han establecido para cumplir con este requisito varias previsiones. Entre las más importantes están los siguientes [5]:

a) La 'residenciación' del estudiante en los servicios clínicos del hospital, en una jornada matutina completa, de lunes a jueves, buscando la total integración del alumno en las actividades clínicas del servicio durante el periodo que dura la rotación (casi tres meses), pasando luego a nuevas rotaciones en otros servicios del hospital.

b) La aparición de tres nuevas asignaturas, denominadas 'Práctica clínica' (I, II y III, a cursar en cuarto, quinto y sexto año del grado), absolutamente prácticas, con una calificación independiente, integradas por 16 módulos de contenido diverso (medicina, cirugía, pediatría, obstetricia, psiquiatría, traumatología y atención primaria), que se desarrollan siguiendo un mapa de competencias lógico, aceptado y diseñado por consenso.

c) El establecimiento de una prueba de evaluación de la competencia objetiva y estructurada (ECOE), de carácter obligatorio, a realizar por los alumnos de sexto curso antes de que terminen sus estudios de medicina.

- Que el hospital reúna las características necesarias para que pueda catalogarse con rigor 


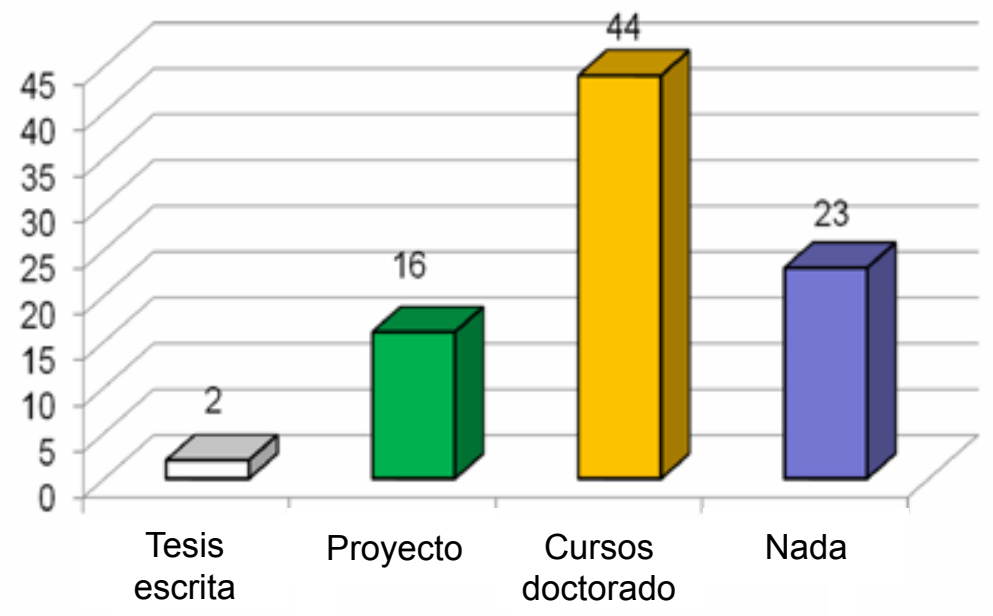

Figura 4. Encuesta transversal, realizada a 82 residentes de aparato digestivo al terminar su periodo de formación, sobre la situación de la tesis doctoral de cada uno de ellos. Puede observarse que sólo en dos casos se había efectuado la tesis doctoral y que en 23 casos ni siquiera se había planteado. Tomado de [7].

como universitario, concepto aún por definir, pero absolutamente imprescindible en el momento actual. Algunas de las propiedades que a este respecto deberían considerarse son:

a) Tener un porcentaje determinado, desde luego no escaso, de doctores entre sus médicos de plantilla.

b) Contar con un número concreto, en ningún caso simbólico, de profesores numerarios de cada especialidad entre los médicos del hospital.

c) Implicar en la enseñanza del grado y del posgrado a todos los médicos de la plantilla mediante la oportuna venia docente, que formaría parte de los derechos y las obligaciones de esos médicos.

d) Tratarse de un hospital general, con una actividad asistencial mínima a determinar (intra y extrahospitalaria) y en el que existan servicios de todas o, al menos, de la mayoría (a establecer) de las especialidades médicas, quirúrgicas y procedimentales.

e) Estar acreditado para la docencia posgraduada.

f) Comprometerse a que la cobertura de las plazas vacantes que se produzcan, al menos las de determinadas categorías médicas, se lleva a cabo mediante un mecanismo abierto, bien definido, transparente y con la publicidad suficiente, que valore, de manera simultánea y ponderada, la capacidad asistencial, docente e investigadora de los candidatos y que asegure, en todo caso, el mérito y la igualdad de oportunidades

g) Considerar a los estudiantes de medicina, al igual que ocurre con los médicos residentes, como una parte integrante del personal del hospital, con las especificaciones o limitaciones que se crean necesarias.

h) Adecuar los 'tiempos médicos' (consultas, pruebas diagnósticas, formas terapéuticas, etc.) a las necesidades docentes reales, es decir, a los requerimientos que exige la enseñanza de los estudiantes de medicina y de los residentes en formación, 'sacrificando' a esta idea, por tanto, unos tiempos que serían menores si sólo se guiaran por la mera asistencia y tuvieran como único objetivo la atención clínica de los enfermos.

Conviene señalar que la ANECA exige actualmente, antes de la verificación de cualquier grado de medicina, que exista una adecuación entre el profesorado y los demás recursos docentes necesarios y la enseñanza que se tiene previsto impartir. Además, hay que recordar que de este organismo no depende el que un hospital se con- 
vierta en universitario. Sin embargo, también es evidente que la adecuación mencionada puede ser, por parte del promotor del proyecto, una simple declaración de intenciones futuras, más o menos factibles.

En todo caso, es evidente que la situación actual, en los hoy en día denominados hospitales universitarios, dista mucho de parecerse, por desgracia y en casi todos los conceptos, a lo que aquí se ha descrito. Ojalá alguna vez se consiga algo que se aproxime conceptual, reglamentaria y estructuralmente a este desiderátum.

\section{Conclusiones}

En atención a lo expuesto en los párrafos anteriores pueden formularse algunas conclusiones, que quizás sean de interés general:

- La formación clínica es, efectivamente, una de las piedras angulares de los estudios de medicina.

- Potenciarla no debe llevar a que se descuide la formación teórica, ni a que se olvide la importancia que también tiene la investigación.

- Para conseguir que la formación clínica sea buena es preciso que se cumplan unos requisitos imprescindibles: que el número de estudiantes de medicina se adecue a las posibilidades docentes reales de cada centro, que los alumnos se incorporen tempranamente a las actividades clínicas de un hospital universitario que merezca tal nombre, y que se disponga de hospitales que reúnan los criterios necesarios para que puedan catalogarse como universitarios en el pleno sentido de la palabra, circunstancia que es absolutamente imprescindible si se quiere mejorar o, al menos, mantener la calidad de la enseñanza de la medicina en España.

\section{Bibliografía}

1. Ministerio de Educación y Ciencia. Real decreto 1393/ 2007, de 29 de octubre (BOE del 30 de octubre de 2007), por el que se establece la ordenación de las enseñanzas universitarias oficiales. BOE 2007; 260: 44037-48.

2. Ministerio de Educación. Real decreto 861/2010, de 2 de julio (BOE del 3 de julio de 2010), por el que se modifica el real decreto 1393/2007, de 29 de octubre, por el que se establece la ordenación de las enseñanzas universitarias oficiales. BOE 2010; 161: 58454-68.

3. Ministerio de Educación y Ciencia. Resolución de la Secretaría de Estado de Universidades e Investigación, del 17 de diciembre de 2007 (BOE del 21 de diciembre de 2007), por la que se publica el acuerdo del Consejo de Ministros de 14 de diciembre de 2007, por el que se establecen las condiciones a las que deberán adecuarse los planes de estudio conducentes a la obtención de títulos que habiliten para el ejercicio de la profesión regulada de médico. BOE 2007; 305: 52849-50.

4. Ministerio de Educación y Ciencia. Orden ECI/332/2008, de 13 de febrero (BOE del 15 de febrero de 2008), por la que se establecen los requisitos para la verificación de los títulos universitarios oficiales que habiliten para el ejercicio de la profesión de médico. BOE 2008; 40: 8351-5.

5. Agencia Nacional de Evaluación de la Calidad y Acreditación. Documento 105216397, del 8 de mayo de 2009, por el que se verifica el plan de estudios del grado en medicina de la Universidad Complutense de Madrid. Madrid: ANECA 2009; 1335: 1-102.

6. Majumdar SR, Roe MT, Peterson ED, Chen AY, Gibler WB, Armstrong PW. Better outcomes for patients treated at hospital that participate in clinical trials. Arch Intern Med 2008; 168: 657-62.

7. Ríos-Zambudio A, Sánchez-Gascón F, González-Moro L, Guerrero-Fernández M. Formación investigadora durante la residencia MIR. Encuesta de satisfacción. Rev Esp Enferm Dig 2004; 96: 700-4. 